\title{
Exact entanglement entropy of the XYZ model and its sine-Gordon limit
}

\author{
Elisa Ercolessi, Stefano Evangelisti and Francesco Ravanini \\ Department of Physics, University of Bologna \\ and I.N.F.N., Sezione di Bologna \\ Via Irnerio 46, 40126 Bologna, Italy \\ ercolessi@bo.infn.it, stafano.evangelisti@gmail.com, ravanini@bo.infn.it
}

\begin{abstract}
We obtain the exact expression for the Von Neumann entropy for an infinite bipartition of the XYZ model, by connecting its reduced density matrix to the corner transfer matrix of the eight vertex model. Then we consider the anisotropic scaling limit of the XYZ chain that yields the $1+1$ dimensional sine-Gordon model. We present the formula for the entanglement entropy of the latter, which has the structure of a dominant logarithmic term plus a constant, in agreement with what is generally expected for a massive quantum field theory.
\end{abstract}

Classification codes (PACS): 02.30.Ik, 11.10.-z, 75.10.Pq, 03.67.Mn, 11.10.-z

Keywords: Integrable spin chains, Integrable quantum field theory, Entanglement in extended quantum systems 


\section{Introduction}

Recently, the investigation of entanglement properties in systems with many degrees of freedom has become a challenging line of research which has put into evidence a growing number of connections between quantum information and computational science, statistical mechanics, quantum field theory as well as formerly very far away topics, such as spin systems and black hole physics.

In particular, much attention has been devoted to the study of strongly correlated spin and/or electronic models in one dimension, with the aim to unveil the relationship between entanglement properties of the ground state and quantum phase transitions. As soon as one has to deal with systems made up of more than two two-level subsystems, there does not exist a unique way of characterizing the degree of entanglement stored by the system itself. Thus, several different measures of entanglement have been proposed in literature over the last few years and used to study the critical properties of the above mentioned systems. These studies include more standard definitions of entanglement measures, such as concurrence [1, 2, 3], as well as others, such as Renyi entropies [4], local [5] indicators, or fidelity [6].

In the realm of quantum field theory, one usually studies entanglement properties of a system via the computation of the so called Von Neumann entropy $S=-\operatorname{Tr}\left[\rho_{A} \log \rho_{A}\right]$ related to the reduced density matrix $\rho_{A}$ of a subsystem A, as proposed by [7], 8] and [9] in the context of black hole physics, and by [10] in that of quantum information. The general theory was then developed by Calabrese and Cardy [11], in which both the critical (conformal) and the free massive cases have been examined. As for the massless situation, much evidence has been accumulated proving that, for a wide class of lattice systems, from the formula for $S$ one can extract the central charge of its scaling limit conformal field theory. This is the case, for example, of spin $\kappa / 2$ XXZ-chains $(\kappa=1,2,3 \ldots)$ [12] or of the $S U(3)$ AFM Heisenberg chain [13]. In addition, some attention has been devoted to the relationship between entanglement properties of the vacuum and the boundary conditions of the theory [11, 12, 14, 15]. Important analytical progress has also been made in the context of massive integrable quantum field models, for which a framework for the computation of Von Neumann entropy has been developed using factorized scattering and form factor techniques [16, 17].

In this paper we will first study the XYZ model and compute the exact expression for the entanglement entropy for an infinite bipartition of such quantum spin chain. As a measure of the entanglement, we will use the Von Neumann entropy of the density matrix associated with a semi-infinite segment of the chain. We will avoid the difficulties concerning the direct 
computation of the density matrix by mapping the quantum chain onto a two-dimensional classical spin system. As Nishino et al. [18, 19] first pointed out, there is a connection between the density matrix of a quantum chain and the classical partition function of a two-dimensional strip with a cut perpendicular to it [20]. In fact, the ground state of the quantum chain described by a Hamiltonian $\hat{H}$ is also an eigenvalue of the row-to-row transfer matrix $T$ of the classical model, provided that $[\hat{H}, T]=0$. In [11], this analogy was used in order to compute the entanglement of a transverse Ising chain and of a XXZ chain near their critical points. By exploiting the existing link between the eight vertex model and the XYZ chain [21, we will obtain a formula for the entanglement entropy for the latter, which once more confirms the following universal expression for $S$, which is valid near criticality when the correlation length $\xi$ is much larger than the lattice spacing $a$ :

$$
S \sim(c / 6) \log (\xi / a)+U
$$

where $c$ is the central charge of the conformal field theory describing the critical point that the theory is approaching. This formula first appeared in [11, but it is a natural consequence of a similar conformal field theory formula first derived by [8, 9]. A similar formula can also be found in [22], but without any mention of the central charge.

Here $U$ is a non-universal constant which is well known to depend on the particular model under investigation. When the bipartition is made up of two semi-infinite chains, as it is in this paper, it has been noted by several authors [11, 12, 14, 15] that it contains information about the so-called Affleck-Ludwig boundary entropy [23].

At the present time, with the method of this paper, we are not able to extract the boundary entropy information from such non-universal $U$, a result that can be achieved only after having calculated the Von Neumann entropy in the finite-length interval case, within the same regularization scheme. Also, the study of the exact link between this term and the boundary entropy needs more accurate calculations on correlation functions that are not yet easily accessible in integrable models. The present exact result, however, could become very useful the day one will be able to compare it with calculations coming from independent methods.

Finally, we will be interested in a particular and relevant scaling limit of the XYZ chain, which yields the $1+1$ dimensional sine-Gordon model [24, 25]. After a brief discussion of the connection between these two models in the thermodynamic limit, we will present the formula for the entanglement entropy of the latter which has a dominant logarithmic term in perfect agreement with what we expected by seeing the sine-Gordon model as a perturbed 
$c=1$ conformal field theory. This formula also gives an analytic expression for the constant $U$.

\section{Entanglement entropy for the XYZ model via corner transfer matrix}

Let us consider the quantum spin- $\frac{1}{2} \mathrm{XYZ}$ chain, which is described by the following hamiltonian

$$
\hat{H}_{X Y Z}=-\sum_{n}\left(J_{x} \sigma_{n}^{x} \sigma_{n+1}^{x}+J_{y} \sigma_{n}^{y} \sigma_{n+1}^{y}+J_{z} \sigma_{n}^{z} \sigma_{n+1}^{z}\right)
$$

where the $\sigma_{n}^{i}(i=x, y, z)$ are Pauli matrices acting on the site $n$, the sum ranges over all sites $n$ of the chain and the constants $J_{x}, J_{y}$ and $J_{z}$ take into account the degree of anisotropy of the model. Without any loss of generality, we can put $J_{x}=1$. In the following we will exploit the very well known connection between the XYZ model and the eight vertex model [26]. Indeed, as shown by Sutherland [21], when the coupling constants of the XYZ model are related to the parameters $\Gamma$ and $\Delta$ of the eight vertex model 1 at zero applied field by the relations

$$
J_{x}: J_{y}: J_{z}=1: \Gamma: \Delta
$$

the row-to-row transfer matrix $T$ of the latter model commutes with the Hamiltonian $\hat{H}$ of the former. It is customary [26] to parametrize the constants $\Delta$ and $\Gamma$ in terms of elliptic functions

$$
\Gamma=\frac{1+k \operatorname{sn}^{2}(i \lambda)}{1-k \operatorname{sn}^{2}(i \lambda)} \quad, \quad \Delta=-\frac{\operatorname{cn}(i \lambda) \operatorname{dn}(i \lambda)}{1-k \operatorname{sn}^{2}(i \lambda)}
$$

where $\operatorname{sn}(x), \operatorname{cn}(x)$ and $\operatorname{dn}(x)$ are Jacobian elliptic functions while $\lambda$ and $k$ are parameters whose domains are the following

$$
0<k<1 \quad, \quad 0<\lambda<I\left(k^{\prime}\right)
$$

$I\left(k^{\prime}\right)$ being the complete elliptic integral of the first kind of argument $k^{\prime}=$ $\sqrt{1-k^{2}}$. We recall that this parametrization is particularly suitable to describe the anti-ferroelectric phase of the eight vertex model, that is when $\Delta<-1$, even if it can be used in all cases by redefining the relations that hold between $\Gamma$ and $\Delta$ and the Boltzmann weights.

\footnotetext{
${ }^{1}$ On the relation among $\Gamma, \Delta$ and the Boltzmann weights of XYZ we adopt here the conventions of [26].
} 
Then, if relation (3) holds, according to [18, 19, 20], in the thermodynamic limit may be obtained the reduced density matrix $\hat{\rho}_{1}$ of the XYZ model relative to a semi-infinite chain as product of the four Corner Transfer Matrices $\hat{A}=\hat{C}$ and $\hat{B}=\hat{D}$ of the eight vertex model at zero field. The starting point is the density matrix defined by $\hat{\rho}=|0\rangle\langle 0|$, where $|0\rangle \in \mathcal{H}$ is the ground state in the infinite tensor product space $\mathcal{H}=\mathcal{H}_{1} \otimes \mathcal{H}_{2}$ of our quantum spin chain. The subscripts ${ }_{1}$ and ${ }_{2}$ refer to the semi-infinite left and right chains. $\hat{\rho}_{1}$ is then defined as the partial trace:

$$
\hat{\rho}_{1}=\operatorname{Tr}_{\mathcal{H}_{2}}(\hat{\rho})
$$

More precisely one can write

$$
\hat{\rho}_{1}\left(\bar{\sigma}, \bar{\sigma}^{\prime}\right)=(\hat{A} \hat{B} \hat{C} \hat{D})_{\bar{\sigma}, \bar{\sigma}^{\prime}}=(\hat{A} \hat{B})_{\bar{\sigma}, \bar{\sigma}^{\prime}}^{2}
$$

where $\bar{\sigma}$ and $\bar{\sigma}^{\prime}$ are particular spin configurations of the semi-infinte chain. Generally speaking, the above quantity does not satisfy the constraint $\operatorname{Tr} \hat{\rho}_{1}=$ 1 , so we must consider a normalized version $\hat{\rho}_{1}^{\prime}$. Let us definine for future convenience the partition function $\mathcal{Z}=\operatorname{Tr} \hat{\rho}_{1}$. For the zero field eight vertex model with fixed boundary conditions, as it is shown in [26, 27, 28], one can write down an explicit form of the Corner Transfer Matrices in the thermodynamic limit

$$
\begin{aligned}
& \hat{A}_{d}(u)=\hat{C}_{d}(u)=\left(\begin{array}{cc}
1 & 0 \\
0 & s
\end{array}\right) \otimes\left(\begin{array}{cc}
1 & 0 \\
0 & s^{2}
\end{array}\right) \otimes\left(\begin{array}{cc}
1 & 0 \\
0 & s^{3}
\end{array}\right) \otimes \ldots \\
& \hat{B}_{d}(u)=\hat{D}_{d}(u)=\left(\begin{array}{ll}
1 & 0 \\
0 & t
\end{array}\right) \otimes\left(\begin{array}{cc}
1 & 0 \\
0 & t^{2}
\end{array}\right) \otimes\left(\begin{array}{cc}
1 & 0 \\
0 & t^{3}
\end{array}\right) \otimes \ldots
\end{aligned}
$$

where $s$ and $t$ are functions of the parameters $\lambda, k$ and $u$ whose explicit expressions are given by $s=\exp [-\pi u / 2 I(k)]$ and $t=\exp [-\pi(\lambda-u) / 2 I(k)]$, $I(k)$ being an elliptic integral of the first kind of modulus $k$. The downscripts ${ }_{d}$ refer to a new basis in which the operators $A$ and $B$ are simultaneously diagonal. We can indeed do that for the present purpose because we are interested in evaluating a trace, hence we are only interested in the eigenvalues of $\rho_{1}$. Thus in this new basis the density operator of formula (7) is given by

$$
\hat{\rho}_{1}=\left(\begin{array}{ll}
1 & 0 \\
0 & x
\end{array}\right) \otimes\left(\begin{array}{cc}
1 & 0 \\
0 & x^{2}
\end{array}\right) \otimes\left(\begin{array}{cc}
1 & 0 \\
0 & x^{3}
\end{array}\right) \otimes \ldots
$$

where $x=(s t)^{2}=\exp [-\pi \lambda / I(k)]$. We notice that $\hat{\rho}_{1}$ is a function of $\lambda$ and $k$ only and that, furthermore, it can be rewritten as

$$
\hat{\rho}_{1}=(\hat{A} \hat{B})^{2}=e^{-\epsilon \hat{O}}
$$


$\hat{O}$ is an operator with integer eigenvalues and

$$
\epsilon=\pi \lambda / I(k)
$$

The Von Neumann entropy $S$ can then be easily calculated according to

$$
S=-\operatorname{Tr} \hat{\rho}_{1}^{\prime} \ln \hat{\rho}_{1}^{\prime}=-\epsilon \frac{\partial \ln \mathcal{Z}}{\partial \epsilon}+\ln \mathcal{Z}
$$

where, in our case, the partition function is given by

$$
\mathcal{Z}=\prod_{j=1}^{\infty}\left(1+x^{j}\right)=\prod_{j=1}^{\infty}\left(1+e^{-\pi \lambda j / I(k)}\right)
$$

Thus we obtain an exact analytic expression for the entanglement entropy of the XYZ model

$$
S_{X Y Z}=\epsilon \sum_{j=1}^{\infty} \frac{j}{\left(1+e^{j \epsilon}\right)}+\sum_{j=1}^{\infty} \ln \left(1+e^{-j \epsilon}\right)
$$

which is valid for generic values of $\lambda$ and $k$. This is the main result of this section. When $\epsilon \ll 1$, i.e. in the scaling limit analogous to the one of [12], formula (14) can be approximated by its Euler-Maclaurin asymptotic expansion, yielding

$$
\begin{aligned}
S_{X Y Z} & =\int_{0}^{\infty} \mathrm{d} x\left(\frac{x \epsilon}{1+e^{x \epsilon}}+\ln \left(1+e^{-x \epsilon}\right)\right)-\frac{\ln 2}{2}+O(\epsilon) \\
& =\frac{\pi^{2}}{6} \frac{1}{\epsilon}-\frac{\ln 2}{2}+O(\epsilon)
\end{aligned}
$$

This will be used in the next subsection where we will check our analytic results with some special known cases, namely the XXZ and the XY chain.

\section{Some checks against known results}

Let us first consider the case $k=0$ (i.e. $\Gamma=1$ ) and the limit $\lambda \rightarrow 0^{+}$, which corresponds to the spin $1 / 2 \mathrm{XXZ}$. In this limit the eight vertex model reduces to the six vertex model. Let us note that formula (15) coincides exactly with the one proposed by Weston [12] which was obtained in the study of more general spin $\kappa / 2$. In this limit the approximation $\epsilon \ll 1$ is still valid and we can use the result of equation (15). From relation (4), it follows that

$$
\lambda=\sqrt{2} \sqrt{-\Delta-1}+O\left((-1-\Delta)^{3 / 2}\right)
$$


Thus equation (15) gives

$$
S=\frac{\pi^{2}}{12 \sqrt{2}} \frac{1}{\sqrt{-\Delta-1}}-\frac{\ln (2)}{2}+O\left((-1-\Delta)^{1 / 2}\right)
$$

which can be written in a simpler form if we recall that, when $\epsilon \rightarrow 0$ (i.e. $\Delta \rightarrow-1^{-}$), the correlation length is given by [26]

$$
\ln \frac{\xi}{a}=\frac{\pi^{2}}{\epsilon}-2 \ln (2)+O\left(e^{-\pi^{2} / \epsilon}\right)
$$

where $a$ is the lattice spacing. Recalling that

$$
\epsilon=2 \sqrt{2} \sqrt{-\Delta-1}+O\left((-1-\Delta)^{3 / 2}\right)
$$

the expression for the entanglement entropy becomes

$$
S=\frac{1}{6} \ln \frac{\xi}{a}+U+O\left((-1-\Delta)^{1 / 2}\right)
$$

where $U=-\ln (2) / 6$. This last expression confirms the general theory of equation (1) with $c=1$, which is exactly what one should expect, the XXZ model along its critical line being a free massless bosonic field theory with $c=1$. The entanglement entropy in XXZ critical chain was also derived from the second law of thermodynamics in [29].

As a second check, we consider the case $\Gamma=0$, which corresponds to the $\mathrm{XY}$ chain. It is convenient now to describe the corresponding eight vertex model by using Ising-like variables which are located on the dual lattice [26], thus obtaining an anisotropic Ising lattice, rotated by $\pi / 4$ with respect to the original one, with interactions round the face with coupling constants $J, J^{\prime}$, as shown in figure 1 .

In our case the Ising lattice decouples into two single sublattices with interactions among nearest neighbors. Now

$$
\Delta=\sinh (2 \beta J) \sinh \left(2 \beta J^{\prime}\right) \equiv k_{I}^{-1}
$$

so that, using the elliptic parametrization, one has

$$
\lambda=\frac{1}{2} I\left(k^{\prime}\right)
$$

Thus $\epsilon$ of equation (11) becomes

$$
\epsilon=\frac{\pi I\left(k_{I}^{\prime}\right)}{I\left(k_{I}\right)}
$$




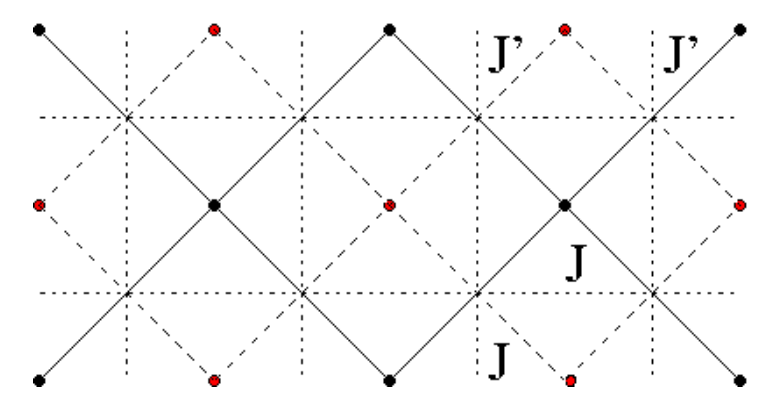

Figure 1: Decoupled anisotropic Ising lattices. Horizontal and vertical lines belong to the original eight vertex model lattice, diagonal lines belong to the dual Ising lattice.

Let us approach the critical line of the anisotropic Ising model from the ferromagnetic phase, i.e. let us assume that $k_{I} \rightarrow 1^{-}$. In this case it is straightforward to write

$$
\epsilon=-\frac{\pi^{2}}{\ln \left(1-k_{I}\right)}+O\left(\ln ^{-2}\left(1-k_{I}\right)\right)
$$

so that the entanglement entropy is

$$
S=-\frac{1}{6} \ln \left(1-k_{I}\right)+O\left(\ln ^{-1}\left(1-k_{I}\right)\right)
$$

Since $\xi^{-1}=\left(1-k_{I}\right)+O\left(\left(1-k_{I}\right)^{2}\right)$, we can easily conclude that

$$
S=\frac{1}{6} \ln \frac{\xi}{a}+O\left(\ln ^{-1}\left(1-k_{I}\right)\right)
$$

where again the leading term confirms the general result (11), with $c=1$. This result is in agreement with what found in previous works [3, 11, 30, 31, 32] by means of different approaches. In particular the results contained in [31, 32] are rigorous mathematical theorems.

\section{The sine-Gordon limit}

In [24, 25] it has been proposed that a particular scaling limit of the XYZ model yields the sine-Gordon theory. In this section we will use this connection to compute the exact entanglement entropy between two semi-infinite intervals of a $1+1$ dimensional sine-Gordon model. In his article, Luther [24] showed that in the scaling limit, where $a \rightarrow 0$ while keeping the mass gap constant, the parameters of the XYZ model and those of the sine-Gordon 
theory are connected by the following relation (keeping $J_{x}=1$ from the beginning)

$$
M=8 \pi\left(\frac{\sin \mu}{\mu}\right)\left(\frac{l_{r}}{4}\right)^{\pi / \mu}
$$

where the parameter $\mu$ is defined as

$$
\mu \equiv \pi\left(1-\frac{\beta^{2}}{8 \pi}\right)=\arccos \left(-J_{z}\right)
$$

Here $M$ is the sine-Gordon solitonic mass, and $l_{r}=l a^{-\mu / \pi}$, with

$$
l^{2}=\frac{1-J_{y}^{2}}{1-J_{z}^{2}}
$$

These relations tell us how the coupling constant $J_{z}$ is connected to the parameter $\beta$ of sine-Gordon, and how $J_{y}$ scales when we take the scaling limit $a \rightarrow 0$. It is clear from equation (29) that in this limit $J_{y} \rightarrow 1^{-}$. In the following we work in the repulsive regime $4 \pi<\beta^{2}<8 \pi$ (which corresponds to $0<\mu<\pi / 2$ and $-1<J_{z}<0$ ). In this regime the mass gap of the theory is the soliton mass $M$. Taking this limit we use the following parametrization of the XYZ coupling constants

$$
\Gamma=\frac{J_{z}}{J_{y}} \quad, \quad \Delta=\frac{1}{J_{y}}
$$

which amounts to a reparametrization of the Boltzmann weights of XYZ suitable for the $|\Delta| \leq 1$ disordered regime where we are working now (see chapter 10 of [26] for details). As a consequence of such reparametrization a minus sign appears in front of both equations (4). Taking the sine-Gordon limit, $\lambda$ and $k$ parametrizing $\Gamma$ and $\Delta$ must now satisfy the following constraint

$$
\operatorname{sn}^{2}(i \lambda)=-\frac{\frac{J_{z}}{J_{y}}+1}{k-k \frac{J_{z}}{J_{y}}}
$$

Considering the parametrization of $\Delta$ and using the properties of the Jacobian elliptic functions we can write

$$
\Delta^{2}=\frac{\operatorname{cn}^{2}(i \lambda) \operatorname{dn}^{2}(i \lambda)}{\left(1-k \operatorname{sn}^{2}(i \lambda)\right)^{2}}=\frac{\left(k\left(1-\frac{J_{z}}{J_{y}}\right)+\frac{J_{z}}{J_{y}}+1\right)\left(k\left(1+\frac{J_{z}}{J_{y}}\right)-\frac{J_{z}}{J_{y}}+1\right)}{4 k}
$$

Expanding around $k \rightarrow 1^{-}$and collecting $\Delta^{2}=1 / J_{y}^{2}$ from both sides of the equation we find

$$
\Delta^{2}=1+\frac{1}{4}\left(1-J_{z}^{2}\right)(k-1)^{2}+O(k-1)^{3}
$$


Using equation (27) we obtain

$$
l^{2}=l_{r}^{2} a^{2 \mu / \pi}=4^{2-3 \mu / \pi}\left(\frac{M \mu a}{\pi \sin \mu}\right)^{2 \mu / \pi}
$$

where $\mu$ is completely fixed by choosing a particular value of $J_{z}$. Now using the definition (29) and (30) we find

$$
\Delta^{2}=1+\left(1-J_{z}^{2}\right) 4^{2-3 \mu / \pi}\left(\frac{M \mu a}{\pi \sin \mu}\right)^{2 \mu / \pi}+O\left(a^{4 \mu / \pi}\right)
$$

which is valid when $a \rightarrow 0$. Comparing equation (33) with (35) we can identify in which way $k$ scales to $1^{-}$

$$
k=1-2^{3(1-\mu / \pi)}\left(\frac{M \mu a}{\pi \sin \mu}\right)^{\mu / \pi}+O\left(a^{2 \mu / \pi}\right)
$$

Remembering the constraint (31) and using the previous expression for $k$ we have

$$
\operatorname{sn}^{2}(i \lambda)=\frac{-J_{z}-1}{1-J_{z}}+O\left(a^{\mu / \pi}\right)
$$

When $k \rightarrow 1$ the elliptic function sn reduces to an hyperbolic tangent, thus we obtain

$$
\tan ^{2} \lambda=\frac{1+J_{z}}{1-J_{z}}+O\left(a^{\mu / \pi}\right) \quad \longrightarrow \quad \lambda=\arctan \sqrt{\frac{1+J_{z}}{1-J_{z}}}+O\left(a^{\mu / \pi}\right)
$$

Now we can evaluate the expression (14) in this limit. Using the following asymptotic behaviour of the elliptic integral $I(x)$

$$
I(x) \approx-\frac{1}{2} \ln (1-x)+\frac{3}{2} \ln 2+O(1-x), \quad x \approx 1^{-}
$$

along with the approximation (15), we can write the exact entanglement entropy of a bipartite XYZ model in the sine-Gordon limit

$$
S_{s G}=-\frac{\pi}{12} \frac{\ln (1-k)-3 \ln 2}{\arctan \sqrt{\frac{1+J_{z}}{1-J_{z}}}}-\frac{\ln 2}{2}+O(1 / \ln (a))
$$

The leading correction to this expression comes from the $O(\epsilon)$ term of equation (15). The constant $J_{z}$ is connected to $\beta$ by

$$
J_{z}=-\cos \pi\left(1-\frac{\beta^{2}}{8 \pi}\right)
$$


thus using this property and the scaling expression (37) we can write down the entanglement entropy as

$$
S_{s G}=\frac{1}{6} \ln \left(\frac{1}{M a}\right)+\frac{1}{6} \ln \left(\frac{\sin \left[\pi\left(1-\frac{\beta^{2}}{8 \pi}\right)\right]}{\left(1-\frac{\beta^{2}}{8 \pi}\right)}\right)+O(1 / \ln (a))
$$

This result confirms the general theory due to [11, 16], in the limit where the system is bipartite in two infinite intervals, with the central charge equal to 1 , as it should, because the sine-Gordon model can be considered a perturbation of a $c=1$ conformal field theory (described by a free massless boson compactified on a circle of radius $\sqrt{\pi} / \beta$ ) by a relevant operator of (left) conformal dimension $\beta^{2} / 8 \pi$. We can write

$$
S_{s G} \approx \frac{1}{6} \ln \left(\frac{1}{M a}\right)+U(\beta) \quad a \rightarrow 0
$$

where the constant term $U(\beta)$ takes the value

$$
U(\beta)=\frac{1}{6} \ln \left(\frac{\sin \left[\pi\left(1-\frac{\beta^{2}}{8 \pi}\right)\right]}{\left(1-\frac{\beta^{2}}{8 \pi}\right)}\right)
$$

At $\beta^{2}=4 \pi$, when the sine-Gordon model becomes the free Dirac fermion theory, it assumes the value $U(\sqrt{4 \pi})=\frac{1}{6} \log 2=0.11552453 \ldots$, while at $\beta^{2}=8 \pi$, where the theory becomes a relevant perturbation of the WZW conformal model of level 1 by its operator of left dimension $\frac{1}{4}$, it becomes $U(\sqrt{8 \pi})=\frac{1}{6} \log \pi=0.19078814 \ldots$

We notice that formula (42) yields the exact value of the overall constant $U(\beta)$, since it has been derived from equation (15) which is exact up to terms $O(\epsilon)$. As mentioned in the introduction and as observed by many authors [7, 8, 10, 19], $U$ should contain a contribution from the Affleck Ludwig boundary term as well as a model-dependent constant that we are not able to extract at this stage. Indeed, this would imply to obtain the Von Neumann entropy for the finite-length interval within the same approach and by using the same (lattice spacing) regularization scheme 2 . In this respect it would be extremely interesting to be able to perform the same calculation in other regularization schemes of the sine-Gordon model. Also, consideration of boundary conditions other than the "vacuum" one considered here could shed light, in the spirit of [12], on the problem of clearly relating this constant

\footnotetext{
${ }^{2}$ This type of calculation can also shed some light on understanding the universal part of the constant $U$ in the case of a massive feld theory, a result that should be then compared with what is known in literature about the conformal case.
} 
to the boundary entropy. In this respect the considerations done in paper [15] are very important. Clearly this question deserves further investigation that we plan for the future.

\section{Conclusions and outlooks}

We have carried out an exact formula for the entanglement entropy in an infinite bipartition of the XYZ model in the thermodynamic limit, by which, as test bench, we have re-obtained some well known results about the entanglement entropy of two integrable systems, the XXZ model and the XY chain.

In addition we have obtained the entanglement entropy of the (repulsive) sine-Gordon scaling limit of the XYZ model, which, on one side, confirms once more the general theory due to [11, 16] and, on the other side, yields for the first time an exact expression for such quantity in the case of an interacting massive field theory. Since we are dealing with a non free theory, an investigation of the connections between this expression, Affleck-Ludwig boundary entropy [23] and one point functions of sine-Gordon fields [33] is non-trivial and deserves further work.

Also it would be important to compare our results with those obtained for massive $1+1$ dimensional theories by the form factor approach of [16, 17] and generalized to any massive (even not integrable) theory in 34. This implies to implement our technique for a subsystem $A$ consisting of a finite interval, a situation that we plan to study in the future.

Finally, let us remark that another issue addressed in 11 is the dependence of entanglement entropy on finite size effects. To investigate this aspect one should implement finite size effects into the XYZ model, possibly within the Bethe ansatz approach, and then rescale to the continuum. A good question to ask is how far this approach can be related to the definition of sine-Gordon model on a cylinder by rescaling lattice models, both in the XYZ approach [35, 36] or in the so called light-cone approach by Destri and De Vega [37. How far the finite size effects on entanglement entropy may be encoded in structures generalizing the non-linear integral equations governing e.g. the finite size rescaling of the central charge $c$ (see e.g. 38 and references therein) and the Affleck-Ludwig boundary entropy $g$ [39, 40, is an intriguing issue, surely deserving further investigation.

All these lines of developments can shed new light on the comprehension of the entanglement problem in quantum field theory. 


\section{Acknowledgments}

We wish to thank Francesco Buccheri, Andrea Cappelli, Filippo Colomo, Marcello Dalmonte, Cristian Degli Esposti Boschi, Benjamin Doyon, Davide Fioravanti, Federica Grestini and Fabio Ortolani for useful and very pleasant discussions. This work was supported in part by two INFN COM4 grants (FI11 and NA41) and by the Italian Ministry of Education, University and Research grant PRIN-2007JHLPEZ.

\section{References}

[1] L. Amico, R. Fazio, A. Osterloch and V. Vedral, Rev. Mod. Phys. 80 (2008) 517 - arXiv:quant-ph/0703044

[2] A. Osterloch, L. Amico, G. Falci and R. Fazio, Nature 416 (2002) 608 - arXiv:quant-ph/0202029

[3] G. Vidal, J. I. Latorre, E. Rico and A. Kitaev, Phys. Rev. Lett. 90 (2003) 227902 - arXiv:quant-ph/0211074

[4] F. Franchini, A. R. Its and V. E. Korepin, J. Phys. A: Math. Theor. 41 (2008) 025302 - arXiv: 0707.2534

[5] L. Campos Venuti, C. Degli Esposti Boschi, G. Morandi, M. Roncaglia and A. Scaramucci, Phys. Rev. A 73 (2006) 010303(R) arXiv:quant-ph/0508236

[6] P. Zanardi and N. Paunković, Phys. Rev. E 74 (2006) 031123 arXiv:quant-ph/0512249

[7] L. Bombelli, R. K. Koul, J. Lee, R. Sorkin, Phys. Rev. D 34 (1986) 373

[8] J. Callan, F. Wilczek, Phys. Lett. B 55 (1994) 333 arXiv:hep-th/9401072

[9] C. Holzhey, F. Larsen, F. Wilczek Nucl. Phys. B 424 (1994) 443 arXiv:hep-th/9403108

[10] C. H. Bennet, H. J. Bernstein, S. Popescu, B. Schumacher, Phys. Rev. A 53 (1996) 2046 - arXiv:quant-ph/9511030

[11] P. Calabrese and J. L. Cardy, J. Stat. Mech. P06002 (2004) arXiv:hep-th/0405152 
[12] R. A. Weston, JSTAT 0603 (2006) L002 - arXiv:math-ph/0601038

[13] M. Aguado, M. Asorey, E. Ercolessi, F. Ortolani and S. Pasini, Phys. Rev. B 79 (2009) 012408 - arXiv:0706.3384

[14] Asorey and Muoz-Castaeda, J. Phys. A 41 (2008) 164043 arXiv:0712.4392 and J. Phys. A 41 (2008) 304004 - arXiv:0803.2553

[15] O. A. Castro-Alvaredo, B. Doyon, J. Stat. Phys. 134 (2009) 105 arXiv:0810.0219

[16] J. L. Cardy, O. A. Castro-Alvaredo and B. Doyon, J. Stat. Phys. 130 (2007) 129 - arXiv:0706.3384

[17] O. A. Castro-Alvaredo and B. Doyon, J. Phys. A41 (2008) 275203 arXiv:0802.4231

[18] T. Nishino, J. $\quad$ Phys. $\quad$ Soc. Japan 64 (1995) $3598 \quad-$ arXiv:cond-mat/9508111

[19] T. Nishino and K. Okunishi, J. Phys. Soc. Japan 66 (1997) 3040 arXiv:cond-mat/9705072

[20] I. Peschel, M. Kaulke and O. Legeza, Ann. Phys. (Leipzig) 8 (1999) 153 - arXiv:cond-mat/9810174

[21] B. Sutherland, J. Math. Phys. 11 (1970) 3183

[22] M. Srednicki, Phys. Rev. Lett. 71 (1993) 666 - arXiv:hep-th/9303048

[23] J. Affleck and A. W. W. Ludwig, Phys. Rev. Lett. 67 (1991) 161

[24] A. Luther, Phys. Rev. B14 (1976) 2153

[25] J. D. Johnson, S. Krinsky, B. M. McCoy, Phys. Rev. A8(1973) 5

[26] R. J. Baxter, Exactly solved models in statistical mechanics, Academic Press, London (1982).

[27] R. J. Baxter, J. Stat. Phys. 15 (1976) 485

[28] R. J. Baxter, J. Stat. Phys. 17 (1977) 1

[29] V. E. Korepin, Phys. Rev. Lett. 92 (2004) - arXiv:cond-mat/0311056

[30] I. Peschel, J. Stat. Mech. P12005 (2004) - arXiv:cond-mat/0410416 
[31] A. R. Its, B. Q. Jin and V. E. Korepin, J. Phys. A38 (2005) 2975 arXiv:quant-ph/0409027

[32] B. Q. Jin, V. E. Korepin, J. Stat. Phys. 116 (2004) 79 arXiv:quant-ph/0304108

[33] S. Lukyanov and A. Zamolodchikov, Nucl. Phys. B 493 (1997) 571 arXiv:hep-th/9611238

[34] B. Doyon, Phys. Rev. Lett. 102 (2009) 031602 - arXiv:0803.1999 [hepth]

[35] D. Fioravanti and M. Rossi, JHEP $0508 \quad$ (2005) 010 arXiv:hep-th/0504122

[36] D. Fioravanti and M. Rossi, Ann. H. Poincar 7 (2006) 1449 arXiv:hep-th/0602080

[37] C. Destri and H. De Vega, Nucl. Phys. B290 (1987) 363

[38] F. Ravanini, Finite size effects in integrable quantum field theories, Lectures given at Eï¡œtvïœœ Summer School in Physics: Nonperturbative QFT Methods and Their Applications, Budapest, Hungary, 14-18 August 2000. Published in "Budapest 2000, Non-perturbative QFT methods and their applications", Horvath Z and Palla L editors, World Scientific, Singapore, (2001) p. 199-264

[39] P. Dorey, D. Fioravanti, C. Rim and R. Tateo, Nucl. Phys. B696 (2004) 445

[40] P. Dorey, A. Lishman, C. Rim and R. Tateo, Nucl. Phys. B744 (2006) 239 